\title{
Excitonic Energy Transfer in Light Emitting Polymers
}

\author{
K. K. Liang ${ }^{a}$, J. H. Hsu ${ }^{\text {a }}$, W. S. Fann ${ }^{a, b}$, S. H. Lin ${ }^{b}$ \\ K. H. Lee ${ }^{\text {c }}$, M. K. Leung ${ }^{\mathrm{c}}$, K. R. Chuang ${ }^{\mathrm{d}}$, and S. A. Chen ${ }^{\mathrm{d}}$ \\ a Physics Department, National Taiwan University, Taipei, Taiwan, R.O.C. \\ ${ }^{b}$ Institute of Atomic and Mrolecular Sciences, Academia Sinica, Taipei, Taiwan, R.O.C. \\ ${ }^{\circ}$ Chemistry Department, National Taiwan University, Taipei, Taiwan, R.O.C. \\ ${ }^{a}$ Chemical Engineering Department, National Tsing Hua University, Hsin Chu, Taiwan, R.O.C.
}

\begin{abstract}
A model including the migration and trapping of excitons on linear chains was proposed to understand the fluorescence dynamics of light emitting polymers. Analytic solution of the model was worked out. To verify the usefulness of the model, timeresolved PL experiments were performed on a series of materials. The characteristics in PL decay dynamics of different materials can be understood with the model qualitatively.
\end{abstract}

Keywords: Photoluminescence, Poly(phenylene vinylene) and derivatives

\section{Introduction}

The photoluminescence (PL) decay in light-emitting. conjugated polymers exhibits non-exponential character. It is generally believed that the quenching of the excitons by defects causes the complicated decay dynamics. The mechanism of cxciton quenching should be important for the designing of materials with better transport properties and quantum efficiency.

In this paper, we proposed a model slightly different from the Balagurov-Vaks model[1] which is often used in analyzing $\mathrm{PL}$ quenching problem[2]. The trapping rate was introduced as a new parameter, and the calculation was done numerically without going to the continuum limit (i.e., the long-chain or the low defect-concentration limit). Experimentally, PL dynamics of materials with and without intrachain transport were used to verify the usefilness of the model.

\section{Theory}

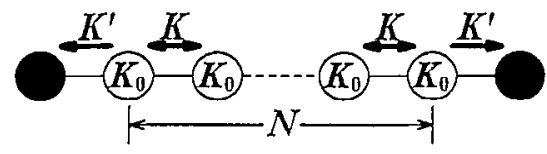

Fig. 1. A conjugated segment between two defect sites. Parameters are explained in text.

The model used in this paper is depicted in Fig. 1. A white spot in Fig. 1, which represents one exciton site, is about six monomer units. The black spots represent the defects on the polymer chain. The natural decay rate (including both natural radiative and non-radiative decay) is $K_{0}$. The migration rate of the exciton on non-defected chain segments is $K$. The defect trapping rate is defined as $K^{\prime}$. The trapning of exciton is assumed to be irreversible. Only nearest neighbor interactions are taken into account. In addition, interchain interaction is not considered in the model. Hence, for a chain segment like that in Fig. 1 (i.e., of length $N$ ), the equation of motion is

$$
\mathbf{W} \cdot \mathbf{P}=d \mathbf{P} / d t
$$

where $\mathbf{P}$ is the $N$-dimensional vector of the exciton population distribution on the $N$ sites; while $\mathbf{W}$ is the Pauli matrix:

$$
\left\{\begin{array}{l}
W_{j j}=-\left(2 K+K_{0}\right)+\left(K-K^{\prime}\right)\left(\delta_{j 1}+\delta_{j N}\right) \\
W_{i j}=K\left(\delta_{j, i+1}+\mathcal{S}_{j, i-1}\right)
\end{array}\right.
$$

The equation can be simplified by Laplace transform or by assuming the exponential form of $\mathbf{P}$. The differential equation is turned into an eigenvalue problem. With proper change of variables the eigenvalue problem is equivalent to solving the following polynomial equation:

$$
d_{N}(X=-\lambda / 2 K)=U_{N}(X)+4 \Delta U_{N-1}(X)+4 \Delta^{2} U_{N-2}(X)=0
$$

where $\Delta \equiv\left(K-K^{\prime}\right) / 2 K$ and $U_{N}$ is the Chebyshev polynomial of the second type. The solutions, which correspond to the $N$ different time constants in the system, can be evaluated without approximation. Subsequently, the eigenvectors corresponding to each eigenvalue can be found. Thereby, special solution of $\mathbf{P}$ corresponding to any initial condition $\mathbf{P}(0)$ can be donle with ease and the PL decay dynamics on this chain segment will be written down in the form of an $N$-exponential decay function.

Fig. 2 shows the calculations for two different values of $N$, corresponding to two different defect densities. Two points should be noticed about the results. First, if $K^{\prime}$ is more than 2 times larger than $K$, then, discard of the value of $N$, the time constarts fall into two groups. The group of faster decay is composed of 2 lifetimes, which are almost identical to each other as $K^{\prime \prime}$ increases. The group of slower decay includes the other $N-2$ lifetimes. These $N-2$ time constants distribute closely together. Thus the decay dynamics for large $K^{\prime}$ is bi-exponential- 
like. For small $K^{\prime}$ the decay curve is multi-exponential-like. Second, in the bi-exponential decay, the relative weight of the fast components depends strongly on defects density, $N$. If $N$ is large, which corresponds to low defect concentration, the slow decay dominate. Because there are only 2 fast components but $N-2$ slow components. This explains why the decay in the pristine samples is exponential. On the contrary, the fast components dominate for small $N$.

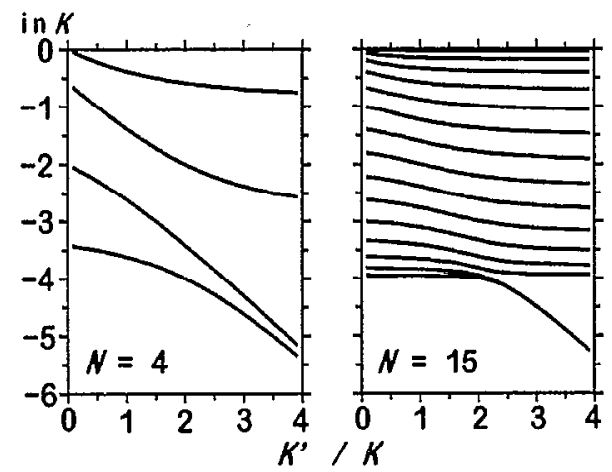

Fig. 2. Characteristic lifetimes w.r.t. the ratio between the trapping rate $K^{\prime}$ and migration rate $K$, for $N=4$ and $N=15$, respectively.

\section{Experiments}

The time-resolved PL spectra were measured with a timecorrelated single photon counting system (TCSPC) based on a Ti:Sapphire mode-locked laser. The time-resolution of TCSPC is around 100 ps.

Three different types of samples were used in the experiments. In order to exclude the inter-chain effects, all materials are prepared as dilute solution in organic solvent.

(1) Oligomeric PPV: The oligomer is trimer of PPV which is composed of 3 benzene ring and 2 vinyl groups. Only natural decay is supposed to be observable in the solutions of these molecules. $K_{0}$ can therefore be estimated.

(2) PPVCOC10: A copolymer, of which the monomer is composed of a conjugated segment similar to the trimer PPV, and

a non-conjugated blocking segment of $\mathrm{O}-\left(\mathrm{CH}_{2}\right)_{10}-\mathrm{O}$. Due to the non-conjugated blocking group in the monomer, it is expected that the excitons created on the conjugated segments can not migrate on the chain. The PL dynamics should be identical to that of a trimer PPV according to the current model. (3) RO-PPV: A soluble PPV derivative. The excitons should be able to migrate freely and trapping by defects should occur on the chain. $K$ and $K^{\prime}$ can be estimated by analyzing its PL data.

\section{Results and Discussion}

The PL dynamics of oligomer and copolymer PPV are shown in Fig. 3. The decay curves can be fittcd well by exponential functions with lifetimes of $1.1 \mathrm{~ns}$ and $1 \mathrm{~ns}$, respectively. The fact that both the oligomer and the copolymer show the same decay dynamics is consistent with the model. The non-exponential decay comes from the migration and trapping of excitons.
The PL decay of RO-PPV is shown in Fig. 4(a), along with a bi-exponential fit to the data. It is natural to expect that the time constants obtained from the fitting can be used to determine the parameters in the model. However, it is found that, with the assumption of homogeneous initial exciton population, the greatest ratio between fast and slow components is $2: 1$, which is much smaller than the 27:1 ratio obtained from fitting in Fig. 4(a). Though by assuming inhomogeneous initial population, any fast-to-slow ratio can be fit. However, there is no justification for using inhomogeneous population, unless it can be proved that the photo-excitations are more likely to form near the defects. A trial fitting is shown in Fig. 4(b) to elucidate the bi-exponential character of the current model, discard of the chain length assumed. Notice the large $K^{\prime} / K$ ratio used.

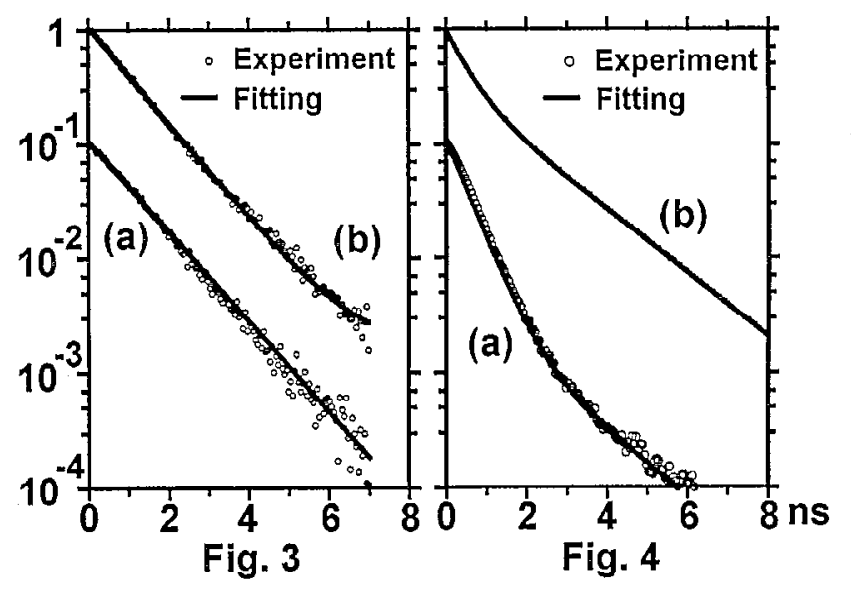

Fig. 3. PL decay of (a) trimer PPV; (b) PPVCOC10. Lifetimes: 1.1 ns and 1.0 ns, respectively.

Fig. 4. (a) PL decay of RO-PPV. Bi-exponential fitting shows $96.5 \%$ of 471 ps lifetime and $3.5 \%$ of $1.56 \mathrm{~ns}$ lifetime. (b) Decay curve evaluated with the current model. $1 / K$ is set to $100 \mathrm{~ns}$. $1 / K^{\prime}$ is set to $666.7 \mathrm{ps} .1 / K_{0}$ is set to $1.61 \mathrm{~ns}$. The resulted dynamics is bi-exponential with $66 \%$ of fast (469 ps) and $34 \%$ of slow (1.563 ns) components. *

In conclusion, the model of particle on a 1-D chain can be used to explain the PL dynamics in light-emitting conjugated polymers qualitatively. The novel feature of the present work is the inclusion of a different trapping rate, which is not adopted by earlier works due to the unsolved numerical complexity. We have removed this difficulty and thus demonstrate the interesting trend of the dynamics when $K^{\prime}$ is somewhat larger than $K$. However, if the initial population is homogeneous, the model can not match the experimental data quantitatively.

\section{References}

[1] B. Ya. Balagurov and V. G. Vaks, Sov. Phys.-JETP, 38 (1974) 968.

[2] M. Yan, L. J. Rothberg, F. Papadimitrakopoulos, M. E. Galvin, and T. M. Miller, Phys. Rev. Lett., 73 (1994) 744. 